\title{
Measurement of human rotation behavior for psychological and neuropsychological investigations
}

\author{
Kaspar Leuenberger · Reto Hofmann · Peter Brugger • \\ Roger Gassert
}

Published online: 15 January 2015

(C) Psychonomic Society, Inc. 2015

\begin{abstract}
The investigation of rotation behavior in human beings enjoys a longstanding and enduring interest in laterality research. While in animal studies the issue of accurately measuring the number of rotations has been solved and is widely applied in practice, it is still challenging to assess the rotation behavior of humans in daily life. We propose a robust method to assess human rotation behavior based on recordings from a miniature inertial measurement unit that can be worn unobtrusively on a belt. We investigate the effect of different combinations of lowcost sensors-including accelerometers, gyroscopes, and magnetometers - on rotation measurement accuracy, propose a simple calibration procedure, and validate the method on data from a predefined path through and around buildings. Results suggest that a rotation estimation based on the fusion of accelerometer, gyroscope, and magnetometer measurements outperforms methods based solely on earth magnetic field measurements, as proposed in previous studies, by a drop in error rate of up to $32 \%$. We further show that magnetometer signals do not significantly contribute
\end{abstract}

K. Leuenberger $(\bowtie) \cdot R$. Gassert

Department of Health Sciences and Technology, ETH Zurich, Leonhardstrasse 27, 8092 Zurich, Switzerland

e-mail: lkaspar@ethz.ch

R. Gassert

e-mail: gassertr@ethz.ch

R. Hofmann

Department of Mechanical and Process Engineering, ETH Zurich,

Zurich Switzerland

P. Brugger

Neuropsychology Unit, Department of Neurology, University Hospital Zurich, Zurich Switzerland to measurement accuracy in short-term measurements, and could thus be omitted for improved robustness in environments with magnetic field disturbances. Results also suggest that our simple calibration procedure can compete with more complex approaches and reduce the error rate of the proposed algorithm by up to $38 \%$.

Keywords Locomotion · Circling behavior · Turning behavior · Lateral bias · Motor system · Neuropsychiatry . Dopamine - Inertial measurement unit - Accelerometer . Gyroscope $\cdot$ Magnetometer $\cdot$ Long-term activity monitoring

\section{Introduction}

In the dawn of "embodied cognition" (Barsalou 2008), the measurement of human whole-body movements has gained increased attention. One important movement characteristic, associated with a vast number of cognitive functions arguably mediated by one or the other cerebral hemisphere, is body rotation, or turning bias. Turning bias has been extensively studied in animal species from amphibians (Rogers 2002) to fish (Vallortigara and Bisazza 2002), and especially in rodents (Glick et al. 1976). The model of the "circling rat" (Glick and Ross 1981) has helped to establish dopaminergic imbalances underlying asymmetric manifestations of Parkinson's disease. It is a robust finding that animals with unihemispheric lesions rotate in the direction of the hemisphere with a lesion-induced dopamine deficiency (Dunnett and Torres 2012).

In healthy human beings, rotation behavior enjoys a longstanding and enduring interest in laterality research. Comprehensive field studies (Schaeffer 1928) 
of "spiral movements in man" documented a left-sided (counterclockwise) preference during long-term locomotion, ruling out a possible role of peripheral asymmetries, such as leg length (Souman et al. 2009). Rather, the preferred direction of rotation was recognized as a marker of (neuropharmacological and cognitive) asymmetries between the two cerebral hemispheres. More recent research has confirmed the association between psychiatric disease and increased counterclockwise rotations (Bracha et al. 1993). On the basis of sound neuropharmacological evidence, it is assumed that both schizophrenic delusions and left-sided body turns are a direct consequence of a hemi-hyperdopaminergia of the right cerebral hemisphere (Bracha 1989). Similarly, Parkinsonian patients with an asymmetric hemispheric dopamine depletion were shown to preferably rotate towards the more affected hemisphere during unconstrained long-term locomotion (Bracha et al. 1987; Patino et al. 1995). Asymmetric locomotion is also observed after unihemispheric stroke. In rodents, ipsilesional rotation occurs after ischemia-induced focal infarction (Ishibashi et al. 2004), whereas in human patients, turning or veering tendencies reportedly depend on the way of ambulation. While walking shows an ipsilesional bias, driving a powered wheelchair led to a bias towards the opposite, contralesional side (Turton et al. 2009). Standardized assessment of extrapyramidal symptoms like veering or rotation behavior would appear desirable, especially in view of providing individually tailored pharmacological treatment (Ishibashi et al. 2004).

A major challenge in such studies is obtaining a reliable measure of rotation behavior over extended periods of time. In animals, methods relying on a human observer (Robinson et al. 1980), automated procedures based on video recordings (Schwarting et al. 1993; Bonatz et al. 1987), as well as mechanical or electrical sensors (Ungerstedt and Arbuthnott 1970; Greenstein and Glick 1975; Heredia-Lopez et al. 2002), have been proposed. The latter solution, often referred to as rotometer, is typically based on a string connected to the animal, which transmits the rotation to a mechanical or electrical counter. The counter records single turns in both directions with a resolution of up to a quarter turn. Field studies investigating rotation behavior in humans during long-term locomotion have made use of the earth's magnetic field captured by sensors integrated into vests that had to be continuously worn by the subjects (Bracha et al. 1987; Bracha et al. 1993; Mohr, Bracha and Brugger 2003; Mohr, Landis, Bracha, Brugger, et al. 2003; Mohr and Lievesley 2007). These devices capture magnetic north relative to the user's orientation using a compass transducer. A microcontroller extracts quarter turns and counts a full turn when four consecutive quarter turns in the same direction are registered. This output logic corresponds to the output generated by tethered rotometers that have been used for rodents (Bracha et al. 1987).

Whereas the methods proposed for animal studies have been validated and found to be robust, magnetic field sensors used in human studies are known to be heavily disturbed inside and near buildings due to large ferrous structures. It is therefore of interest to characterize the influence of magnetic disturbances on the counts in human rotation behavior studies. Furthermore, previous studies did not document the hardware and algorithms that were used for rotation counting, thus limiting a reproduction and reuse of these systems and methods in future studies.

Here, we present a method to track rotation behavior in humans using a miniature wearable inertial measurement unit (IMU) that was specifically designed for long-term activity and motion monitoring, and complements magnetic field sensing with accelerometer and gyroscope measurements. We propose a simple calibration method for the inertial and magnetic field sensors, and compare rotationtracking performance based solely on magnetometer data to MARG (magnetic, angular rate, and gravity) and IMU measurement fusion methods. We show that counting turns of a human using gyroscopes, accelerometers, and magnetometers reduces the error rate by up to $32 \%$ over the course of a 15-min track (approximately 1,000 m) leading through and around buildings, compared to a system which only relies on magnetic field measurements. The proposed algorithms are robust to slight body sway and orientation changes as they appear in daily tasks such as opening a door. The developed algorithm can be adapted for use with nearly any IMU, both for applications requiring offline or even online processing, as all tasks only depend on data from the past and the mathematical operations can be performed in real time by any modern microcontroller. Although the required heading information could also be extracted from the trajectory estimated by a foot-mounted pedestrian tracking system (e.g., (Foxlin 2005)), our method omits the estimation of the foot trajectory, thus making the approach simpler and probably less prone to errors, as dead reckoning is much more sensitive to precise sensor calibration.

This manuscript is organized as follows: first, the used hardware is introduced, followed by a description of the calibration method and rotometer algorithm, as well as the validation method; we then present results from nine subjects on a predefined track through and around buildings, and discuss the implications of our findings for future studies aiming to assess human rotation behavior. 


\section{Materials and methods}

\section{ReSense}

We used a watch-sized, low-power 10-degrees-of-freedom (DOF) IMU for the purpose of precisely recording human posture and motion data during daily life for time spans that are unreached by other systems with a comparable formfactor. The sensor module can continuously record data for at least $24 \mathrm{~h}$ at a sampling rate of $50 \mathrm{~Hz}$, and this time span can be doubled by means of an intelligent power management (Leuenberger and Gassert 2011).

The inertial unit is comprised of a three-axis accelerometer (ADXL345, Analog Devices), a three-axis gyroscope (ITG-3050, Invensense) and a three-axis magnetometer (MAG3110, Freescale). Furthermore, a barometric pressure sensor (BMP085, Bosch) is integrated to track changes in altitude. The sensor readings can be logged to an internal microSD card, while an internal real-time clock provides absolute timestamps with millisecond resolution. The electronics board is encased in a robust, water-resistant plastic housing. ReSense weighs $15 \mathrm{~g}$ (including the battery and housing) and measures $36 \times 29 \times 13 \mathrm{~mm}^{3}$. A base station (Fig. 1a) allows easy data readout, time synchronization of multiple modules, modification of system parameters, and loading of firmware updates.

\section{Rotometer algorithm}

\section{Calibration}

Low-cost microelectromechanical systems (MEMS) sensors suffer from various errors such as bias and scale factor variations. These errors can be described by the following, simplified model:

$s_{m}=K \cdot s_{t}+b$

where $s_{m}$ is the measured quantity, $s_{t}$ the true quantity, $b$ the sensor bias, and $K$ the scaling factor. Any influence of external temperature changes is neglected in this work and the measurements are assumed to be performed at constant room temperature.

A simple three-step calibration method is proposed to estimate and correct for $K$ and $b$ of the accelerometer and gyroscope and $b$ of the magnetometer. The sensor is attached to a cube, aligned with one of the faces, and the cube is placed on a flat horizontal surface, such that gravity acts perpendicular to the surface (Fig. 2). The calibration method then consists of (i) statically placing the cube in six known orientations (6th orientation on the reverse side of the horizontal surface); (ii) performing exactly ten full rotations
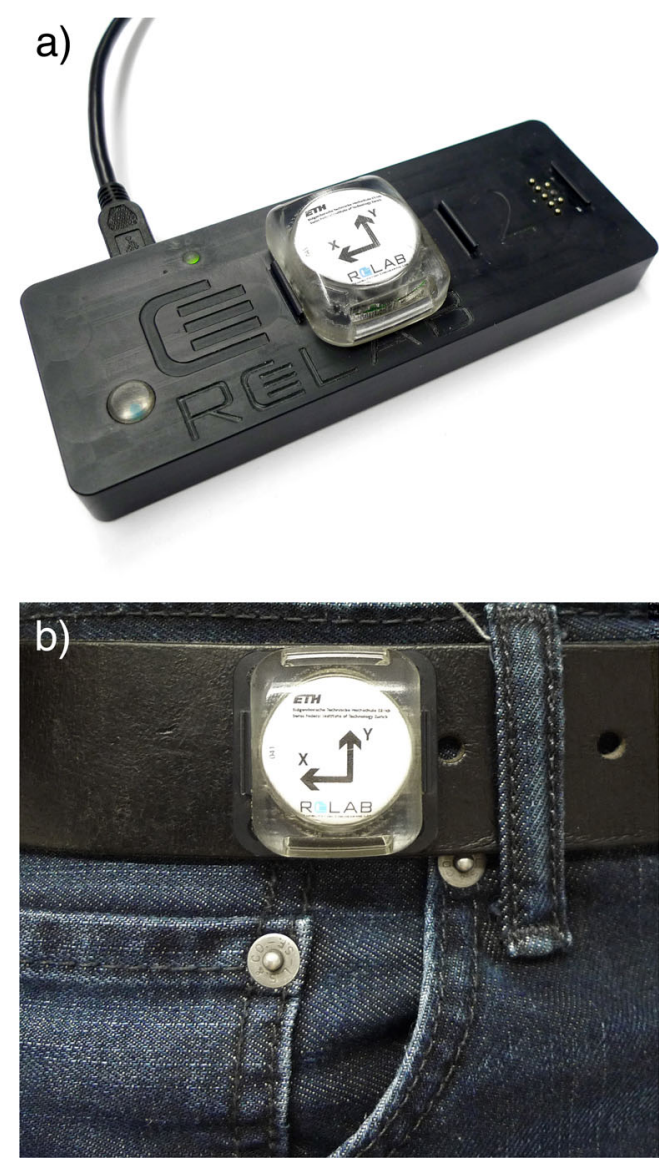

Fig. 1 a) A ReSense sensor module inserted into the base station. b) The sensor module mounted on a belt with a clip system

in both directions around each axis, by hand on the leveled surface; and (iii) freely rotating the cube in space for about $15-20 \mathrm{~s}$.

To assure that, in the second step, the cube was rotated by a multiple of exactly $360^{\circ}$, the sensor module was aligned with an edge before and after the rotations. The accelerometer bias and scale factors were estimated based on the six static measurements using the general least squares approach (Titterton and Weston 2004; Syed et al. 2007). The gyroscope bias and scale factor were estimated from the data set containing exactly ten rotations around each axis in both directions. The bias was calculated by taking the average zero offset when no motion was present. For each axis, the bias-corrected angular velocity was numerically integrated using the trapezoidal rule to calculate the total angle $\delta \theta_{x}, \delta \theta_{y}, \delta \theta_{z}$ of the ten rotations:

$\delta \theta_{x, y, z}=\frac{1}{F_{s}} \sum_{n=1}^{N} \omega_{x, y, z}[n]-b$ 


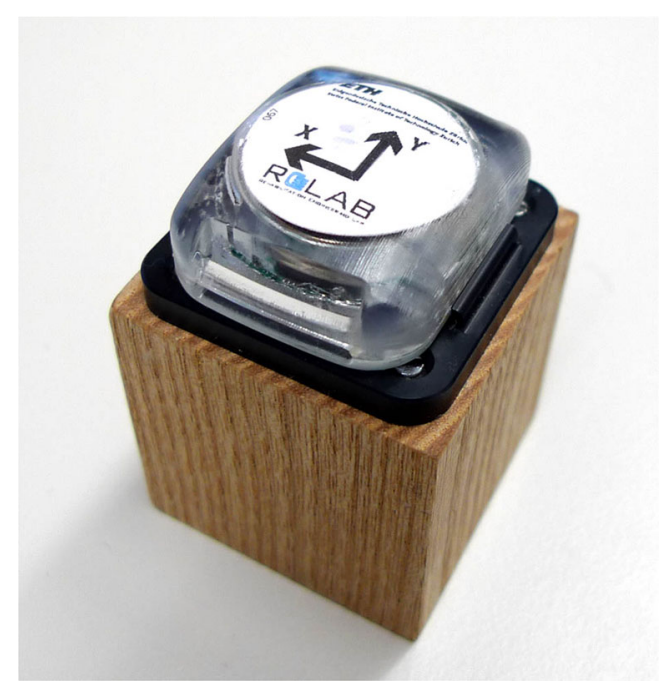

Fig. 2 A ReSense sensor module attached to the calibration cube for manual calibration

where $N$ is equal to the sample number after ten full turns and $F_{S}$ is the sampling frequency. Again, the general least squares approach was used to calculate the scale coefficients based on the angles $\delta \theta_{x, y, z}$.

The magnetometer bias was estimated from the dataset containing the measurements with the free rotations of the cube in space. We used the least squares approach proposed by the magnetometer manufacturer (Ozyagcilar 2012). Any soft-iron effects were neglected and the sensitivity error was not estimated because of lack of a precise reference magnetic field.

\section{Computation of the subject's yaw angle}

To compute the yaw angle, the calibrated inertial and magnetometer measurements were fused to estimate the orientation of the ReSense sensor module relative to the earth frame. For this purpose, a gradient descent orientation filter with a quaternion representation as proposed by (Madgwick et al. 2011) was selected. The filter takes the raw sensor measurements from the gyroscope, accelerometer, and magnetometer and fuses them into an optimal orientation estimate (MARG fusion; magnetic, angular rate and gravity) by compensating for integral drift and assuring convergence from the initial conditions. The filter requires only a single adjustable parameter, $\beta$, which represents the gyroscope measurement error. According to (Madgwick et al. 2011), the optimal value is $\beta=0.03$, and was adopted in this work. The filter can also be run on inertial data only (IMU configuration; gravity and angular rate) with the disadvantage of not being able to account for drift and initial orientation offset in the horizontal plane.
To count the number of turns and thereby assess the rotation behavior of a subject, the rotations around the axis formed by the intersection of the sagittal and the coronal plane are of interest (z-axis, cf. Fig. 3). This is equal to counting the rotations around the yaw axis in the Euler angle representation of the output of the orientation filter. A drawback of using Euler angle representations is the singularities at $\pm 90^{\circ}$, which cause problems as soon as a subject lies down and thus tilts the sensor into a singularity. To resolve this problem, a different approach for calculating the yaw angle (also heading) is introduced. A set of 12 heading vectors $\mathbf{h}_{\mathbf{0}}^{\mathbf{b}}, \mathbf{h}_{\mathbf{1}}^{\mathbf{b}}, \ldots, \mathbf{h}_{\mathbf{1 1}}^{\mathbf{b}}$ is defined in the transverse plane, which are equally spaced by an angle $\varphi$, e.g., $\mathbf{h}_{\mathbf{0}}^{\mathbf{b}}=\left[\begin{array}{lll}1 & 0 & 0\end{array}\right]$, (cf. Fig. 3). These vectors are rotated into the earth frame using the quaternions $\mathbf{q}$ obtained from the orientation filter:

$\mathbf{q}=\left[q_{0}, q_{1}, q_{2}, q_{3}\right] \hat{\mathbf{h}}_{n}^{b}=\left[0, \mathbf{h}_{\mathbf{n}}^{\mathbf{b}}\right]$

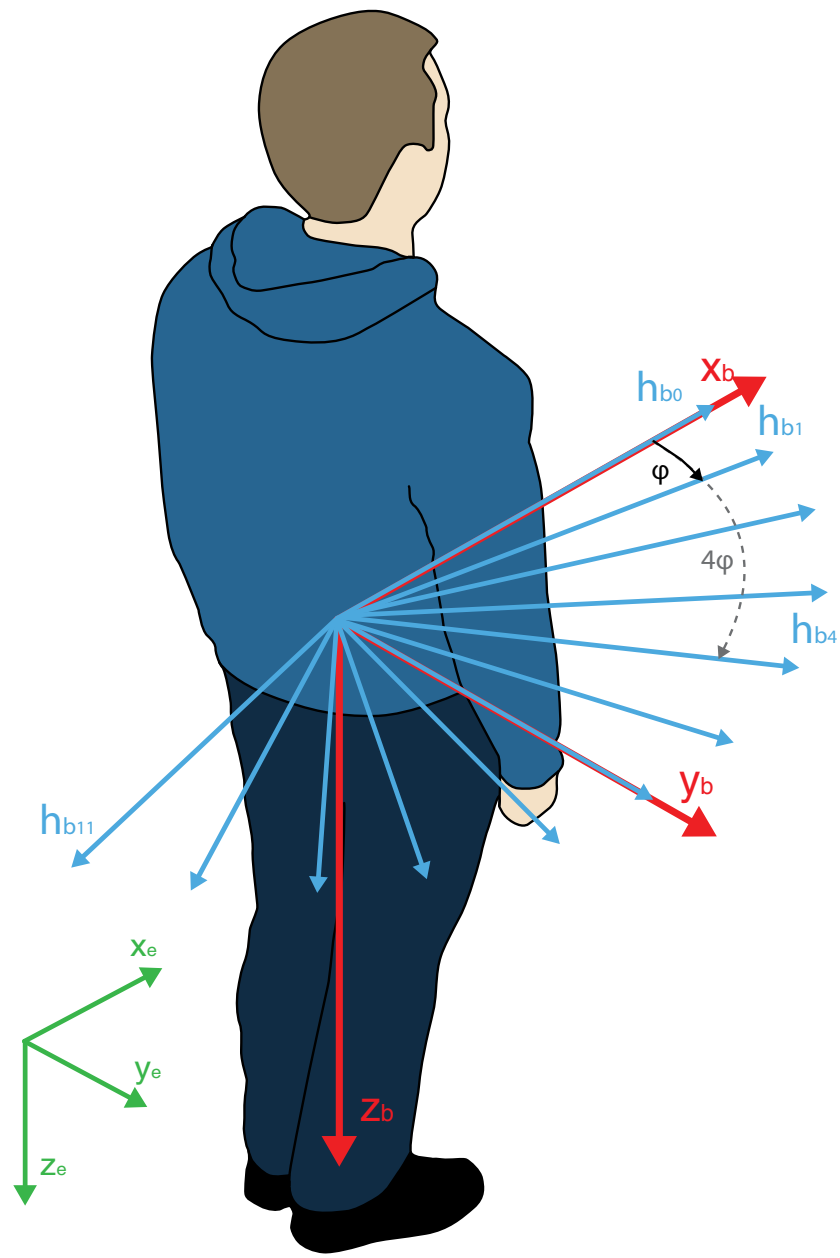

Fig. 3 Heading vectors (blue, $\mathbf{h}_{\mathbf{0}}^{\mathbf{b}}, \mathbf{h}_{\mathbf{1}}^{\mathbf{b}}, \ldots, \mathbf{h}_{\mathbf{1 1}}^{\mathbf{b}}$ ) in the horizontal plane of the body frame, equally spaced by $\varphi$. These vectors are introduced to overcome the limitations due to singularities. The yaw angle of the subject in the earth frame $e$ (also heading) can be determined independent of its 3D orientation by using the vector $\mathbf{h}_{\mathbf{n}}^{\mathbf{e}}$ with the smallest vertical component $z_{e}$ 
$\hat{\mathbf{h}}_{n}^{e}=\mathbf{q} \otimes \hat{\mathbf{h}}_{n}^{b} \otimes \overline{\mathbf{q}}$

where $\otimes$ denotes the quaternion product and $\overline{\mathbf{q}}$ is the conjugate of $\mathbf{q}$.

The yaw angle of the subject can then be calculated as $\operatorname{atan} 2\left(h_{n y}^{e}, h_{n x}^{e}\right)$. Like the Euler angles, the $\operatorname{atan} 2$ function has a singularity for either one of its components equal to zero. To determine which vector $\mathbf{h}_{\mathbf{n}}^{\mathbf{e}}$ should be used to calculate yaw, the one with the smallest $z_{e}$-component is chosen. Its initial offset $n \cdot \varphi$ is corrected prior the calculation of the yaw angle. The sensor module may now be tilted in any direction; there will always be a vector $\mathbf{h}_{\mathbf{n}}^{\mathbf{e}}$ which remains close to the horizontal plane and thus is not prone to causing a singularity. When the module is tilted more than $\pm 90^{\circ}$, the direction of the subject is reversed, and thus the sign of the $z_{e}$-component has to be taken into account when calculating the number of turns.

\section{Counting individual turns}

To be able to determine a possible bias in a human's turning behavior, the number of individual turns in both directions must be counted. The counts can be extracted from the timecourse of the absolute trunk orientation in a way similar to what has been done in rodent studies: the individual rotations are counted in both directions for quarter, half, three-quarter and full turns. A rotation is registered when the angle reaches a predefined threshold without returning through a hysteresis window (Fig. 4). The amount of hysteresis increases with the rotation level and was empirically defined as $20^{\circ}, 40^{\circ}, 60^{\circ}$ and $80^{\circ}$ for $90^{\circ}, 180^{\circ}, 270^{\circ}$ and $360^{\circ}$ turns respectively. To allow for the simultaneous

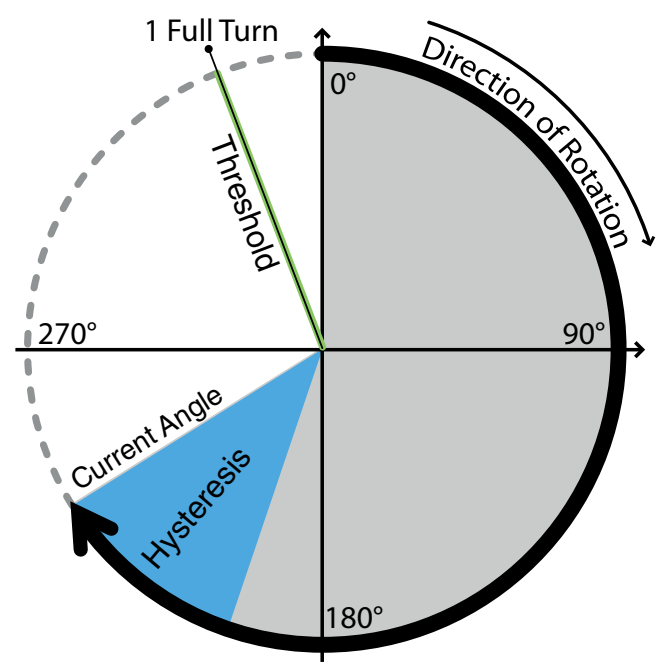

Fig. 4 Example of a turn in the clockwise direction. A full rotation is registered when the angle reaches the green zone without ever inverting all the way through the blue hysteresis zone, which follows the current angle during monotonic increase counting of different rotation levels and apply individual thresholds and hystereses for each level, the procedure is split into eight independent counters (four levels for both directions). The threshold for a valid rotation was defined as ExpectedRotation $-10^{\circ}$ for all levels, where Expected Rotation can be $90^{\circ}, 180^{\circ}, 270^{\circ}$ or $360^{\circ}$. The angle of the individual turn is reset to zero once the rotation is completed or the hysteresis is traversed in the reverse direction. The introduction of a threshold was necessary, as directional changes during locomotion can rarely be divided into exact $90^{\circ}$ segments. As an example, walking in a corridor composed of one $90^{\circ}$ right turn followed by a $90^{\circ}$ left turn will likely be subjected to an optimization by the subject, comprised of a straight line from the right corner of the first turn to the left corner of the second turn, thus resulting in two turns of slightly less than $90^{\circ}$. By introducing a hysteresis before resetting the direction of the turn, the algorithm becomes more robust against noise and parasitic movements, such as slight oscillation of the trunk during normal gait.

\section{Validation}

\section{Calibration method}

The method used to calibrate the MEMS accelerometers is well described in the literature (Titterton and Weston 2004; Syed et al. 2007) and was therefore not validated in this work.

The proposed manual gyroscope gain calibration method was compared to a method using a rate table with a constant angular velocity, which is a commonly used approach to calibrate MEMS gyroscopes. A direct drive system composed of a DC motor (RE40, Maxon Motors, Switzerland) powered by a servo amplifier (ADS 50/5, Maxon Motors, Switzerland) in combination with a high-resolution rotary incremental encoder (R137, Gurley Precision Instruments, USA) with 720,000 counts per revolution was used as a calibration bench (Fig. 5). The control was accomplished using LabVIEW (National Instruments, USA) running at $1 \mathrm{kHz}$ on a real-time target PC system. Constant angular rates of $\pm 500^{\circ} / \mathrm{s}$ and $\pm 1000^{\circ} / \mathrm{s}$ (SD $\left.\pm 0.5 \%\right)$ could be achieved and were used to individually excite the $\mathrm{X}, \mathrm{Y}$, and $\mathrm{Z}$ axis of the gyroscope. A least squares approach was used to estimate the reference scale and bias error. In total, six repetitions of both calibration methods were performed within $1 \mathrm{~h}$ at a temperature of approximately $23^{\circ} \mathrm{C}$. The complete rate table procedure was repeated twice, 2 weeks apart, in order to identify temporal changes in the gyroscope sensitivity. The two distinct methods were each executed six times with one single sensor module and the outcomes were compared with a two-sample $t$ test after reassurance of a normal distribution with the Shapiro-Wilk test $(p<0.05)$. 


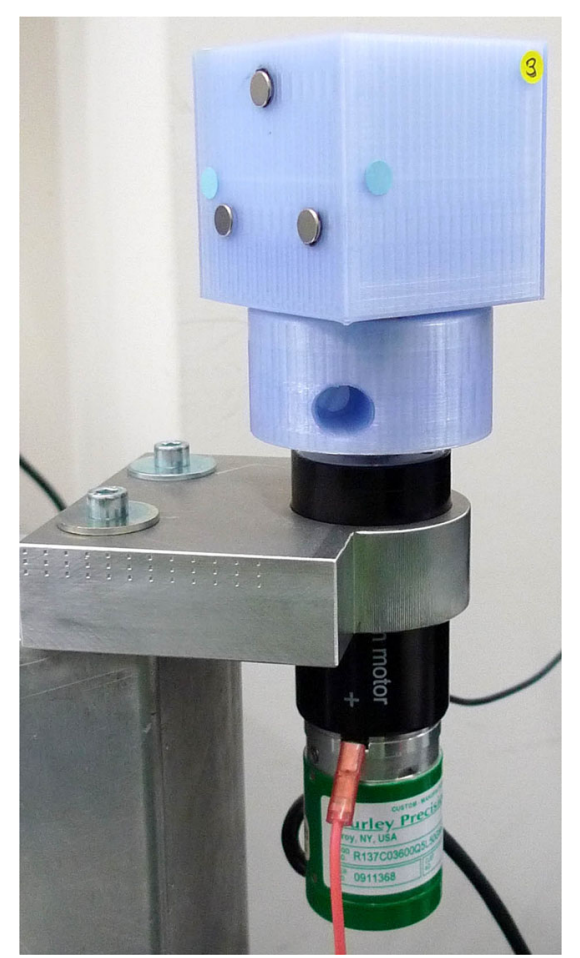

Fig. 5 Setup to spin the sensor module at a constant angular rate. The cube on top carries the sensor module and can be positioned in three different orientations. It is held in place by permanent magnets inserted into the faces of the cube as well as into the base element on the motor shaft

A validation of the magnetometer calibration method was not performed due to the lack of a well-defined and uniform magnetic field that could be used as a reference.

\section{Counting algorithm}

To validate the developed algorithm, nine subjects (seven male, two female, age $27.0 \pm 3.3$ ) completed a predefined path of about 1,000 m around the ETH Zurich central campus (Fig. 6). The path lead through indoor and outdoor areas, contained stair ascents and descents, and involved the opening and closing of doors, an action which was shown to result in body rotation independent of the path. A total of nine runs (one per subject) were performed, where in eight runs the subjects were equipped with a single ReSense sensor module (the same sensor module was used for all subjects) mounted on the belt (Fig. 1b) in order to validate the algorithm, and in one run an additional subject carried six sensor modules simultaneously in a pocket. Data from the last subject were analyzed separately as we were interested in the variability of the results across the six sensor modules. The six sensors were mounted on a cardboard sheet and carried in a trouser pocket in order to show that additional movement artifacts do not negatively affect the rotation measurements, and that all sensors provide similar results. The execution time of each run was approximately $15 \mathrm{~min}$, and all data were sampled continuously at $50 \mathrm{~Hz}$. One of the subjects was filmed from behind along the complete path using a miniature camera (GoPro Hero2, Woodman Labs, USA).

Data processing and analysis were performed offline using MATLAB 8.3 (The MathWorks, USA). Using a map of the predefined trajectory and the recorded videos as reference, the expected heading of a subject walking along the path was determined. The counting algorithm was then applied on this reference data set in order to extract a reference profile of left and right turns with their respective timing. According to the reference path, the following number of turns are expected: 34R/45L @ 90, $13 \mathrm{R} / 17 \mathrm{~L} @$ $180^{\circ}, 7 \mathrm{R} / 11 \mathrm{~L} @ 270^{\circ}$ and 4R/7L @ 360 $0^{\circ}$. In order to correct for different and variable walking speeds, the heading profile of each subject (based on the MARG algorithm) was aligned with the reference dataset using Dynamic Time Warping (Berndt and Clifford 1994) before the counting procedure. Each counting event generated from the recorded motion path was then classified as true or false with respect to the reference dataset. A counting event was labeled as true when a turn in the given direction was present in the reference dataset around that specific time point. It was labeled as false when no turn in the given direction was present in the reference dataset around that time (false positive) or when a turn was missed in the measurement (false negative). Also, the order of the sequence of left/right turns had to match the order of the reference. In other words, the comparison of recorded turns with the reference was treated as a classification task, rather than only comparing final counts with the reference values. The error rate was calculated as a performance measure of each run according to the following equation:

$$
\text { Error Rate }=\frac{\sum \text { turns }_{\text {false }}}{\sum \text { turns }_{\text {false }}+\sum \text { turns }_{\text {true }}}
$$

The output of the counting algorithm using four different sensor combinations for orientation estimation (using the Madgwick fusion algorithm (Madgwick et al. 2011)) was evaluated and the performance was compared with a Wilcoxon rank-sum test as the presence of a normal distribution in the dataset could not be guaranteed (Shapiro-Wilk test $(p<0.05))$. In order to detect a potential bias in counting left or right turns, the means of left and right turn error rate were also compared with a Wilcoxon rank-sum test.

The effect of the following sensor combinations for orientation estimation was investigated in this work:

MARG algorithm based on an optimal fusion of calibrated accelerometer, gyroscope, and magnetometer measurements. 


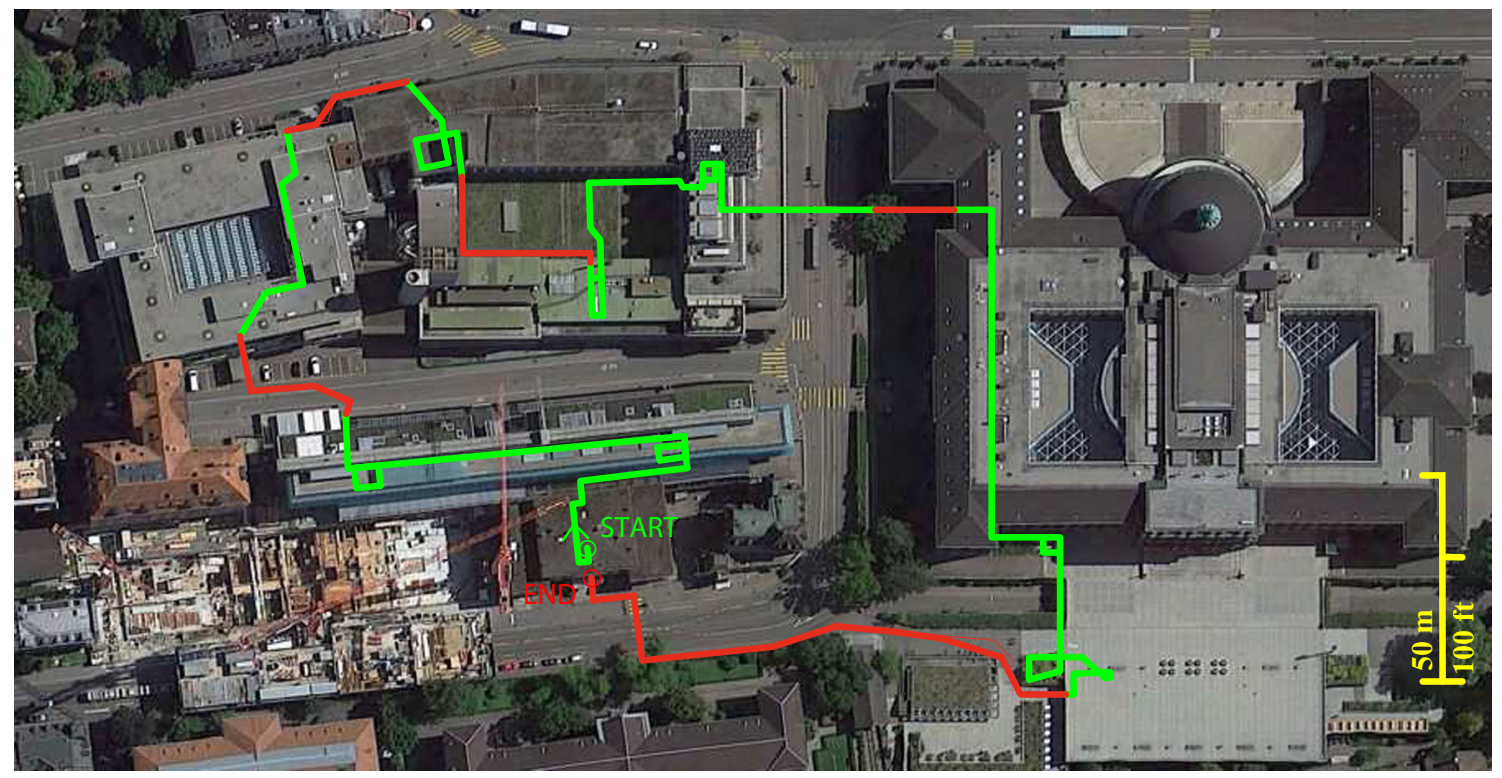

Fig. 6 Map of the traversed path on the ETH Zurich central campus used for validation. Green and red lines denote segments located inside/outside of buildings, respectively. The path is about $1,000 \mathrm{~m}$ long and took subjects about $15 \mathrm{~min}$ to complete Image adapted from Google Maps

IMU

algorithm based on an optimal fusion of calibrated accelerometer and gyroscope measurements.

IMU RAW algorithm based on an optimal fusion of uncalibrated accelerometer and gyroscope measurements.

MAG algorithm based on accelerometer and magnetometer measurements (obtained by running the orientation filter with an angular rate input forced to zero and a $\beta$ of 1.0 to allow for fast convergence of gravity and magnetic field vectors.

\section{Results}

The average scale error factor and bias of the six calibration trials using the two calibration methods are listed in Table 1. While the estimated biases for the individual axes differed significantly between the two methods, this was not the case for the estimated scale factor error $(p<0.05)$. The largest standard deviation of the scale factor error of the manual calibration approach reached $0.122 \%$, whereas the rate table approach stayed within $0.002 \%$. Differences of up to $0.22 \%$ were found between samples of the two scale factor estimation methods. A repetition of the rate table calibration procedure 2 weeks after the first calibration resulted in a difference of $0.02 \%$.

The error rates of the rotometer algorithm based on the four different sensor fusion methods are illustrated in Fig. 7, and the corresponding values are listed in Table 2 . The eight subjects, which carried one sensor module, walked the path in $14.3 \mathrm{~min} \pm 1.0 \mathrm{~min}$. For turns of $90^{\circ}$, the MARG and IMU sensor fusion algorithms resulted in the lowest error rates ( $13.90 \%$ and $14.29 \%$, respectively). The overall error rate of MAG was $29.90 \%$ and that of IMU RAW $29.52 \%$. None of the methods showed significant differences in error rate between left and right turns $(\mathrm{p}<0.05)$. In the case of $180^{\circ}$ turns, the MARG and IMU methods produced the lowest error rate, with $6.67 \%$ and $7.62 \%$, respectively. The MAG error rate was $24.29 \%$ and the IMU RAW had an error rate of $29.05 \%$. Error rates for MAG and IMU RAW showed a significant bias towards left turns. Turns of $270^{\circ}$ were counted with error rates of $4.51 \%, 8.27 \%, 25.56 \%$, and $36.09 \%$ (MARG, IMU, MAG, and IMU RAW). All methods differed significantly in left and right turn error rates; MARG and IMU with a bias towards the right, and MAG and IMU RAW with a bias towards the left. Looking at full

Table 1 Average gyroscope calibration factors (SD in brackets) for the three axes derived from the manual calibration method (Man.) and the rate table method (Mot.)

\begin{tabular}{llll}
\hline & $\mathbf{X}$ & $\mathbf{Y}$ & $\mathbf{Z}$ \\
\hline Scale Err. Man. [\%] & $0.56(0.085)$ & $0.60(0.082)$ & $0.89(0.122)$ \\
Scale Err. Mot. [\%] & $0.55(0.001)$ & $0.64(0.002)$ & $1.00(0.002)$ \\
Bias Man. [\%] & $2.38(0.015)$ & $-0.99(0.010)$ & $-0.09(0.016)$ \\
Bias Mot. [\%] & $2.28(0.004)$ & $-0.64(0.006)$ & $-0.13(0.003)$
\end{tabular}

While the estimated biases for the individual axes differ significantly between the two methods, this is not the case for the estimated scale factor error $(p<0.05)$ 


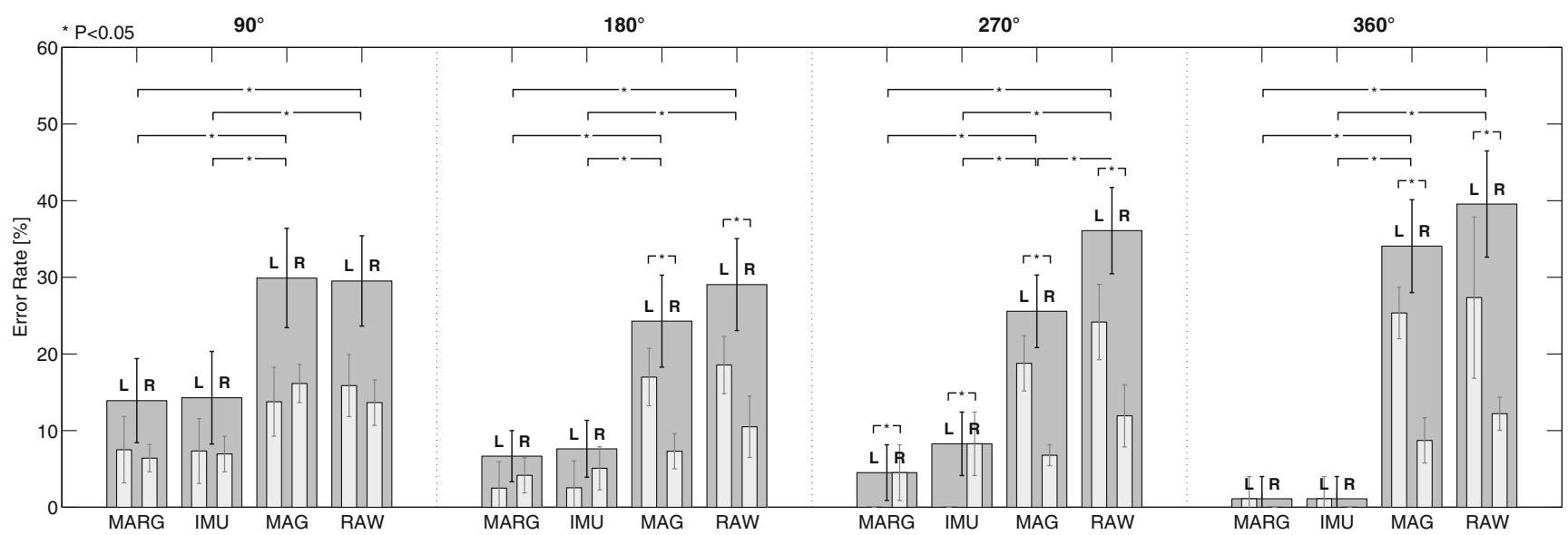

Fig. 7 Performance of different sensor fusion methods in counting turns of $90^{\circ}, 180^{\circ}, 270^{\circ}$ and $360^{\circ}$ : MARG (accelerometer, gyroscope, and magnetometer, calibrated), IMU (accelerometer and gyroscope, calibrated), MAG (magnetometer only, calibrated) and RAW (IMU with uncalibrated sensor data). The dataset consists of eight runs $(n=8)$ performed by eight different subjects. The dark grey bars represent the mean error rate of the miscounted turns of the runs compared to the ideal path extracted from the map (error bars represent standard deviation), whereas the light grey bars show the percentage of left and right mean error rate (normalized within each bar) turns of $360^{\circ}$, the MARG and IMU algorithms both showed error rates of $1.10 \%$. MAG and IMU RAW produced error rates of $34.07 \%$ and $39.56 \%$, and showed a significant bias towards left turns.

Table 3 summarizes the error rates of turn counts for a subject walking the predefined path with six sensor modules simultaneously. The mean error rates were lower than the results presented in the experiment where eight subjects walked along the path, and the standard deviation was $1.01 \%$ in the case of $90^{\circ}, 2.04 \%$ for $270^{\circ}$ and $0.0 \%$ in the other two cases. A comparison of the measured angular profiles showed a maximum difference of up to $77.9^{\circ}(0.22$ turns) and a RMS error of $18.3^{\circ}$ (0.051 turns) over the complete trial. After approximately $15 \mathrm{~min}$, at the end of the trial, the angles calculated from the data of the six modules were within a span of $7.3^{\circ}$.

\section{Discussion}

The results from the trials with eight subjects walking along the predefined path show that the rotometer algorithm utilizing the MARG and IMU orientation filters can accurately count a human's turns while walking indoors and outdoors in a daily setting with error rates little as $1.10 \%$ for $360^{\circ}$ turns. In the experiment, these two methods showed very similar performance across all rotation levels and no significant differences could be found, while the magnetometer-only (MAG) and uncalibrated IMU (IMU RAW) solution showed significantly lower performance. In addition, the two latter showed a direction bias, displaying higher error rates for turns to the left side and rotation levels of $180^{\circ}$ and more. For levels of $270^{\circ}$, MARG and IMU displayed a higher error rate for right turns. We suppose that

Table 2 Error rate of the counting algorithm when using different fusion methods for the orientation filter

\begin{tabular}{|c|c|c|c|c|c|c|c|c|c|c|c|c|c|c|c|c|}
\hline & $90^{\circ}$ & & & & $180^{\circ}$ & & & & $270^{\circ}$ & & & & $360^{\circ}$ & & & \\
\hline & MARG & IMU & MAG & RAW & MARG & IMU & MAG & RAW & MARG & IMU & MAG & RAW & MARG & IMU & MAG & RAW \\
\hline L turns & $\begin{array}{l}15.15 \\
(8.75)\end{array}$ & $\begin{array}{l}14.72 \\
(8.47)\end{array}$ & $\begin{array}{l}27.27 \\
(8.92)\end{array}$ & $\begin{array}{l}32.03 \\
(8.18)\end{array}$ & $\begin{array}{l}4.76 \\
(6.56)\end{array}$ & $\begin{array}{l}4.76 \\
(6.56)\end{array}$ & $\begin{array}{l}36.90 \\
(8.13)\end{array}$ & $\begin{array}{l}39.29 \\
(7.93)\end{array}$ & $\begin{array}{l}0.00 \\
(0.00)\end{array}$ & $\begin{array}{l}0.00 \\
(0.00)\end{array}$ & $\begin{array}{l}42.86 \\
(8.25)\end{array}$ & $\begin{array}{l}53.06 \\
(10.80)\end{array}$ & $\begin{array}{l}2.86 \\
(7.56)\end{array}$ & $\begin{array}{l}2.86 \\
(7.56)\end{array}$ & $\begin{array}{l}57.14 \\
(7.56)\end{array}$ & $\begin{array}{l}60.00 \\
(23.09)\end{array}$ \\
\hline R turns & $\begin{array}{l}12.93 \\
(3.60)\end{array}$ & $\begin{array}{l}13.95 \\
(4.65)\end{array}$ & $\begin{array}{l}31.97 \\
(4.93)\end{array}$ & $\begin{array}{l}27.55 \\
(5.97)\end{array}$ & $\begin{array}{l}7.94 \\
(4.37)\end{array}$ & $\begin{array}{l}9.52 \\
(5.28)\end{array}$ & $\begin{array}{l}15.87 \\
(5.00)\end{array}$ & $\begin{array}{l}22.22 \\
(8.49)\end{array}$ & $\begin{array}{l}7.14 \\
(5.75)\end{array}$ & $\begin{array}{l}13.10 \\
(6.56)\end{array}$ & $\begin{array}{l}15.48 \\
(3.15)\end{array}$ & $\begin{array}{l}26.19 \\
(8.91)\end{array}$ & $\begin{array}{l}0.00 \\
(0.00)\end{array}$ & $\begin{array}{l}0.00 \\
(0.00)\end{array}$ & $\begin{array}{l}19.64 \\
(6.68)\end{array}$ & $\begin{array}{l}26.79 \\
(4.72)\end{array}$ \\
\hline Total turns & $\begin{array}{l}13.90 \\
(5.49)\end{array}$ & $\begin{array}{l}14.29 \\
(6.05)\end{array}$ & $\begin{array}{l}29.90 \\
(6.48)\end{array}$ & $\begin{array}{l}29.52 \\
(5.88)\end{array}$ & $\begin{array}{l}6.67 \\
(3.33)\end{array}$ & $\begin{array}{l}7.62 \\
(3.71)\end{array}$ & $\begin{array}{l}24.29 \\
(6.00)\end{array}$ & $\begin{array}{l}29.05 \\
(6.00)\end{array}$ & $\begin{array}{l}4.51 \\
(3.63)\end{array}$ & $\begin{array}{l}8.27 \\
(4.14)\end{array}$ & $\begin{array}{l}25.56 \\
(4.74)\end{array}$ & $\begin{array}{l}36.09 \\
(5.63)\end{array}$ & $\begin{array}{l}1.10 \\
(2.91)\end{array}$ & $\begin{array}{l}1.10 \\
(2.91)\end{array}$ & $\begin{array}{l}34.07 \\
(6.05)\end{array}$ & $\begin{array}{l}39.56 \\
(6.92)\end{array}$ \\
\hline
\end{tabular}

The numbers represent the mean error rate (in percent) of counted turns of the eight runs compared to the ideal path extracted from the map (standard deviation in brackets) 
Table 3 Average error rate (in percent) of detected turns using six sensor modules carried simultaneously by one subject (standard deviation in brackets)

\begin{tabular}{lllll}
\hline & $\mathbf{9 0}^{\circ}$ & $\mathbf{1 8 0}^{\circ}$ & $\mathbf{2 7 0}^{\circ}$ & $\mathbf{3 6 0}^{\circ}$ \\
\hline L turns & $4.29(1.56)$ & $7.14(0.0)$ & $0.00(0.0)$ & $0.00(0.0)$ \\
R turns & $3.99(0.89)$ & $11.11(0.0)$ & $1.39(3.4)$ & $0.00(0.0)$ \\
Total turns & $4.12(1.01)$ & $9.38(0.0)$ & $0.83(2.04)$ & $0.00(0.0)$ \\
\hline
\end{tabular}

The MARG algorithm was used for orientation estimation

this is due to the fact that the predefined path is more likely to produce errors in right turns for $270^{\circ}$, as the subjects had to cope with doors that caused a rotation towards the left and could thus result in a reset of the $270^{\circ}$ counter within an expected $270^{\circ}$ turn. These rotations during door opening were not captured by our "ground truth", which was based on the expected turns derived from the map.

The low performance of the magnetometer-only approach was expected, as magnetometers are known to be unreliable close to large ferrous installations and high voltage lines as well as inside large buildings such as train stations, parking garages, and shopping malls, etc. One must therefore assume that the results of previous studies, which were based on this technology and performed in critical environments, were affected by such artifacts.

The computed turns deviate significantly from the ground truth when using uncalibrated sensor data to estimate the yaw angle. This is not surprising considering the bias and sensitivity errors from which low-cost MEMS sensors suffer. The results indicate that even with a simple calibration procedure, the influence of these errors can be drastically reduced (by more than $38 \%$ in our example). However, when looking at the results from the trial in which a subject wore six sensors simultaneously, it becomes clear that not all inaccuracies could be eliminated through calibration, and variations between multiple sensors can still develop.

In general, we identified the action of opening a door as an important error source on the predefined path. This action can heavily affect the orientation of the body, which is typically rotated while pulling the door open. The amount of rotation, however, varies between subjects, and in some cases the door was opened by another person walking before the subject or in the opposite direction. Thus, in some trials, these movements were counted as quarter turns and eventually completed a half, three-quarter or full turn. Also, subjects had to avoid dynamic obstacles such as a moving car or other pedestrians, which introduced some variability between trials and could not be controlled for in the validation as no ground truth was available for this experiment. The latter would have required an optical tracking system or similar along the entire path. Also, video recordings only allow an estimation of the rotation angles, but not a precise measurement. Furthermore, they were not available for all participants. Small differences in the calculated yaw angle, which are close to the predefined thresholds but only cross this threshold in some of the cases, can also lead to variability in the number of turns. These factors can explain the measured variations in the eight subjects. The predefined thresholds in the algorithm might lead to a slightly higher number of counted turns, whereas the added hysteresis makes the algorithm more robust. However, the higher the chosen hysteresis, the lower the ability to detect directional changes. All the measurements were conducted with the same sensor module, therefore we can exclude the influence of sensor bias and sensitivity errors, as well as how these two were corrected for in the calibration process as a possible source of variability in the measurements between subjects.

The results from the two calibration methods show differences in the gyroscope bias estimation. Yet, these differences are small and in the worst case the residual bias would add up to one full turn in 17 min of walking along a straight line, which is largely above the timescale of turns in a daily setting. In contrast, performing no bias calibration could lead to one full turn of drift within one minute of straight walking. We suppose that this explains the reason for the biased error rates between left and right turns for the IMU RAW algorithm. The residual low frequency drift after calibration is too slow to be visible in the results of the IMU algorithm, as a subject's directional changes in the experiment happen at a rate of about six $90^{\circ}$ turns per minute. As the algorithm looks at relative angular rotations and not at absolute values it acts as a high-pass filter, thus masking low-frequency influences. We expect that this masking effect might also apply for a daily setting, where a human rarely walks straight for extended periods of time. In case the drift should turn out to be an issue for longer recordings, we suggest implementing a bias correction by measuring the average zero-rate output during phases where the sensor is motionless, and then subtracting this offset from subsequent measurements. Also, the residual drift is low enough to be compensated by the magnetometer in the MARG fusion method, and so this approach is less prone to producing biased results. Magnetometers, however, require regular calibration as magnetization of ferrous materials in a sensor module can vary rather quickly. In contrast, accelerometers and gyrosco pes require less frequent calibration, which is favorable for a user-friendly application.

The results also show that the simple manual calibration approach could approximate the gyroscope scale factor error. However, the precision of the manual approach was poor compared to using a rate table and, thus, in a single calibration run, considerable deviations from the correct scale factor could arise. In a worst-case scenario derived from our calibration measurements, these deviations could lead to a 
$0.8^{\circ}$ error after a $360^{\circ}$ turn. An uncalibrated gyroscope in contrast could lead to $3.5^{\circ}$ error. For an application such as the rotometer, this error can still be considered acceptable, as other factors such as individual gait patterns dominate in inducing error in the overall algorithm. With a precise rate table used for calibration, the gyroscope gain error could be kept in a range of less than $0.08^{\circ}$ over a $360^{\circ}$ turn, even considering temporal changes. However, this is an accuracy that is not required for the rotometer.

Lastly, we have to mention some limitations of this study. The course was predefined and rather short and will thus likely not capture all problems and sources of interference that can occur in a daily setting. Longer recordings in a daily environment, however, would require the subject to be followed with a camera, which then requires intensive labeling of the video files and is also prone to errors as the exact angles of turns can hardly be extracted from video recordings. Moreover, the number of subjects included in the validation of the algorithm is small and the results and conclusions drawn from this data would have to be confirmed in a larger population. Further, the sensor calibration assumes constant temperature conditions, although the temperature of the environment of the sensor module may vary, thus inducing additional drift in the measurements. However, as the sensor is carried close to the body with a constant temperature, we can expect that such variations are limited.

\section{Conclusions}

This paper presented an unobtrusive method to reliably measure rotation behavior of humans and count their turns in a similar way to what has been done in animal models. While prior art exists, previous studies did not report on the used algorithms and relied mostly on magnetometer readings, which are known to be influenced by magnetic disturbances present in and around buildings, as also demonstrated by our investigations. We proposed a simple calibration procedure for the inertial and magnetic sensors that can be implemented with little hardware investment and an algorithm that can be applied with nearly any IMU. We could demonstrate that the fusion of magnetic field measurements with inertial sensor measurements, respectively relying purely on inertial sensors, is more reliable for this application than using magnetic measurements only. For the measurement of rotation behavior in human subjects, the results suggest to use either MARG or IMU fusion methods. We suggest to use MARG only when a complete calibration of the magnetometer is performed by a trained person, and to apply the IMU fusion method otherwise. Thanks to its small size and weight, our rotometer can unobtrusively be worn on a belt and it is well suited for conducting long-term studies during daily life, especially also in neurological patients with ambulation difficulties. The IMU can be used to detect periods of ambulation (Leuenberger et al. 2014; MoncadaTorres et al. 2014), and only these segments can then be analyzed in order to exclude the rotations due to other means of mobility, such as riding a car. Combined with the simple calibration method and the proposed sensor fusion algorithm, this results in a powerful tool for the investigation of rotation behavior in psychological and neuropsychological studies.

Acknowledgments The authors would like to thank the subjects who participated in the study. This work was supported by a private foundation and the National Center of Competence in Research on Neural Plasticity and Repair of the Swiss National Science Foundation. Peter Brugger and Roger Gassert are members of the Neuroscience Center Zurich (ZNZ), Roger Gassert is member of the Rehabilitation Initiative and Technology Platform Zurich (RITZ) and Peter Brugger of the University Zurich Center for Integrative Human Physiology (ZIHP).

\section{References}

Barsalou, L.W. (2008). Grounded cognition. Annual Review of Psychology, 59(1), 617-645.

Berndt, D.J., \& Clifford, J. (1994). Using dynamic time warping to find patterns in time series. In KDD workshop, (Vol. 10 pp. 359-370).

Bonatz, A.E., Steiner, H., Huston, J.P. (1987). Video image analysis of behavior by microcomputer: categorization of turning and locomotion after 6-ohda injection into the substantia nigra. Journal of Neuroscience Methods, 22(1), 13-26.

Bracha, H.S. (1989). Is there a right hemi-hyper-dopaminergic psychosis Schizophrenia Research, 2(4), 317-324.

Bracha, H.S., Livingston, R.L., Clothier, J., Linington, B.B., Karson, C.N. (1993). Correlation of severity of psychiatric patients delusions with right hemispatial inattention (left-turning behavior). American Journal of Psychiatry, 150, 330-332.

Bracha, H.S., Seitz, D.J., Otemaa, J., Glick, S.D. (1987). Rotational movement (circling) in normal humans: sex difference and relationship to hand, foot and eye preference. Brain Research, 411(2), 231-235.

Dunnett, S.B., \& Torres, E.M. (2012). Rotation in the 6-ohda-lesioned rat. In Animal models of movement disorders, (Vol. 61 pp. 299 $315)$.

Foxlin, E. (2005). Pedestrian tracking with shoe-mounted inertial sensors. IEEE Computer Graphics and Applications, 25(6), 3846.

Glick, S., Jerussi, T., Fleisher, L. (1976). Turning in circles: the neuropharmacology of rotation. Life Sciences, 18(9), 889-896.

Glick, S.D., \& Ross, D.A. (1981). Lateralization of function in the rat brain: basic mechanisms may be operative in humans. Trends in Neurosciences, (Vol. 4 pp. 196-199).

Greenstein, S., \& Glick, S. (1975). Improved automated apparatus for recording rotation (circling behavior) in rats or mice. Pharmacology Biochemistry and Behavior, 3(3), 507-510.

Heredia-Lopez, F., Bata-Garcia, J., Alvarez-Cervera, F., GongoraAlfaro, J. (2002). A novel rotometer based on a RISC microcontroller. Behavior Research Methods. Instruments, \& Computers, 34(3), 399-407.

Ishibashi, S., Kuroiwa, T., Katsumata, N., Yuan, S., Endo, S., Mizusawa, H. (2004). Extrapyramidal motor symptoms versus 
striatal infarction volume after focal ischemia in Mongolian gerbils. Neuroscience, 127(2), 269 ?275.

Leuenberger, K., \& Gassert, R. (2011). Low-power sensor module for long-term activity monitoring. In Engineering in medicine and biology society, embc, 2011 annual international conference of, pages $=2237-2241$,

Leuenberger, K., Gonzenbach, R., Wiedmer, E., Luft, A., Gassert, R. (2014). Classification of stair ascent and descent in stroke patients. In Wearable and implantable body sensor networks (BSN), 2014 eleventh international conference on (p. In press.)

Madgwick, S.O., Harrison, A.J., Vaidyanathan, R. (2011). Estimation of IMU and MARG orientation using a gradient descent algorithm. In International Conference on Rehabilitation robotics (ICORR), 2011 IEEE (pp. 1-7).

Mohr, C., Bracha, H.S., Brugger, P. (2003). Magical ideation modulates spatial behavior. The Journal of Neuropsychiatry and Clinical Neurosciences, 15(2), 168-174.

Mohr, C., Landis, T., Bracha, H., Brugger, P., et al. (2003). Opposite turning behavior in right-handers and non-right-handers suggests a link between handedness and cerebral dopamine asymmetries. Behavioral Neuroscience, 117(6), 1448-1452.

Mohr, C., \& Lievesley, A. (2007). Test?retest stability of an experimental measure of human turning behaviour in right-handers, mixed-handers, and left-handers. Laterality, 12(2), 172-190.

Moncada-Torres, A., Leuenberger, K., Gonzenbach, R., Luft, A., Gassert, R. (2014). Activity classification based on inertial and barometric pressure sensors at different anatomical locations. Physiological Measurement, 35(7), 1245-1263.

Ozyagcilar, T. (2012). Calibrating an eCompass in the presence of hard and soft-iron interference.

Patino, P., Garcia-Munoz, M., Freed, C.R. (1995). Electrophysiology of ventromedial striatal neurons during movement. Brain Research Bulletin, 37(5), 481-486.
Robinson, T.E., Becker, J.B., Ramirez, V. (1980). Sex differences in amphetamine-elicited rotational behavior and the lateralization of striatal dopamine in rats. Brain Research Bulletin, 5(5), 539-545.

Rogers, L.J. (2002). Lateralization in vertebrates: its early evolution, general pattern, and development. In Peter, C.T.S., Slater Jay, J.B., Rosenblatt, S., Roper, T.J. (Eds.), (Vol. 31 pp. 107-161): Academic Press.

Schaeffer, A. (1928). Spiral movement in man. Journal of Morphology, 45(1), 293-398.

Schwarting, R., Goldenberg, R., Steiner, H., Fornaguera, J., Huston, J. (1993). A video image analyzing system for open-field behavior in the rat focusing on behavioral asymmetries. Journal of Neuroscience Methods, 49(3), 199-210.

Souman, J.L., Frissen, I., Sreenivasa, M.N., Ernst, M.O. (2009). Walking straight into circles. Current Biology, 19(18), 1538-1542.

Syed, Z., Aggarwal, P., Goodall, C., Niu, X., El-Sheimy, N. (2007). A new multi-position calibration method for MEMS inertial navigation systems. Measurement Science and Technology, 18(7), 1897.

Titterton, D., \& Weston, J. (2004). Strapdown inertial navigation technology, 2nd edition. The Institution of Engineering and Technology, London, United Kingdom and The American Institute of Aeronautics, Reston, Virginia, USA.

Turton, A.J., Dewar, S.J., Lievesley, A., O?Leary, K., Gabb, J., Gilchrist, I.D. (2009). Walking and wheelchair navigation in patients with left visual neglect. Neuropsychological Rehabilitation, 19(2), 274-290.

Ungerstedt, U., \& Arbuthnott, G.W. (1970). Quantitative recording of rotational behavior in rats after 6-hydroxy-dopamine lesions of the nigrostriatal dopamine system. Brain Research, 24(3), $485-493$.

Vallortigara, G., \& Bisazza, A. (2002). How ancient is brain lateralization. Comparative vertebrate lateralization. 